LA-UR- $93-4410$

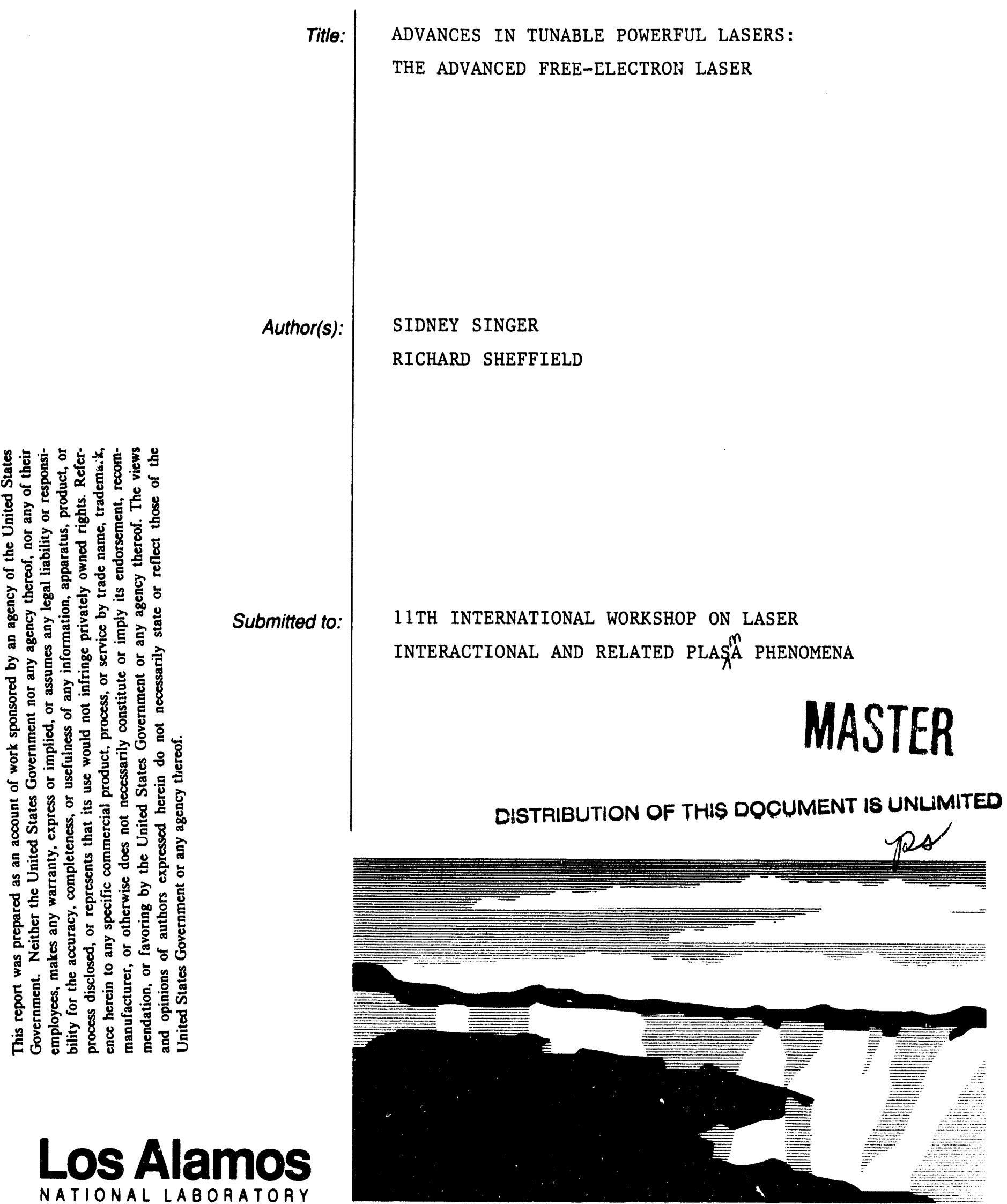

Los Alamos National Laboratory, an affirmative actionvequal opportunity empldyer, is operated by the University of California for the U.S. Department of Eneroy under contract W-7405-ENG-36. By acceptance of this article, the publisher recognizes that the U.S. Government retains a nonexclusive, royalty-free license to publish or reproduce the published form of this contribution, or to allow others to do so, for U.S. Goverrment purposes. The Los Alamos National Laboratory requests that the publisher identity this anticle as work performed under the auspices of the U.S. Department of Energy 


\title{
ADVANCES IN TUNABLE POWERFUL LASERS: THE ADVANCED FREE-ELECTRON LASER
}

\author{
Sidney Singer and Richard Sheffield \\ Los Alamos National Laboratory \\ Los Alamos, NM 87545
}

In the past several decades, remarkable progress in laser science and technology has made it possible to obtain laser light from the ultra-violet to the far infra-red from a variety of laser types, and at power levels from milliwatts to kilowatts (and, some day, megawatts). However, the availability of tunable lasers at "high" power (above a few tens of watts) is more limited. Figure 1, an assessment of the availability of tunable lasers, shows the covered range to be about 400 to 2000 nanometers. A variety of dye lasers cover the visible and near infra red, each one of which is tunable over approximately a $10 \%$ range. In the same region, the TI:saphire laser is adjustable over a 20 to $25 \%$ range. And finally, optical parametric oscillators can cover the range from about 400 nanometers out to about $2000 \mathrm{~nm}$ (even farther at reduced energy output). The typical output energy per pulse may vary from a few to one hundred millijoules, and since repetition rates of 10 to $100 \mathrm{Hertz}$ are generally attainable, average output powers of tens of watts are possible.

In recent years, a new approach to powerful tunable lasers -- the Free-Electron Laser (FEL) -- has emerged. In this paper we will discuss advances in FEL technology which not only enable tunability at high average power over a very broad range of wavelengths, but also make this device more usable. At present, that range is about one micron to the far infra red; with extensions of existing technology, it should be extendable to the vacuum ultra violet region.

Figure 2 shows the basic FEL. Usually, a modulator -- which contains an electron gun -- generates a relatively long (nanoseconds) pulse of electrons which is injected into the buncher. The buncher is an rf accelerator cavity that operates out of phase with the electron beam so as to induce a velocity spread (high velocity at the rear of the beamlet) in the beam. Given a suitable drift path, this results in electron beamlets which are bunched into highercurrent pizosecond-duration pulses. After removing the induced energy spread, the pulses are fed to an accelerator which raises the electron beam energy to an appropriate level. Through the use of magnets the electron beam is deflected and sent to an undulator where the beam is made to wiggle in the undulating spatial magnetic field. This process results in emission of light by the electrons which is trapped between the mirrors of the optical resonator. As the intensity of the light trapped in the resonator increases, its interaction with the beamlets causes them to become bunched on the scale of the optical wavelength, and enables the possibility of significant energy extraction from the electron beam. After leaving the undulator, the electron beam is sent to a beam dump, and the light is out-coupled and conveyed to the area of application. Thus, the traditional FEL uses a thermionic electron gun and long buncher, which requires a great deal of space in order to obtain the bunching, and results in a beam whose emittance is rather high due to space charge induced divergence in the flight path. Low emittance is a critical feature for efficient operation, compactness, and short 
wavelength production of light in an FEL. The normalized emittance $\varepsilon$ is related to the focusability of the electron beam and is defined by

$$
\varepsilon=4 \pi \beta \gamma\left[\left\langle x^{2}\right\rangle\left\langle x^{\prime 2}\right\rangle-\left\langle x x^{\prime}\right\rangle^{2}\right]^{0.5}
$$

where $\boldsymbol{\gamma}$ is the relativistic factor, $\boldsymbol{\beta}$ is the electron velocity divided by the speed of light, $\mathrm{x}$ is the transverse beam size, and $x^{\prime}$ is its divergence.

The equation that defines the wavelength $\lambda_{0}$ of the optical radiation from an FEL is

$$
\lambda_{0}=\frac{\lambda_{w}}{2 \gamma^{2}}\left[1+\frac{K^{2}}{2}\right] \text {, }
$$

where $\lambda_{w}$ is the wiggler period, $\gamma$ is the relativistic energy factor, and $\mathrm{K}$ is the undulator parameter whose value is of order 1 , and implies several options for tuning FELs. First, it is possible to adjust gamma alone to obtain a continuous tuning range of about $20 \%$ of the operating wavelength. If in addition the beam steering and focusing elements are adjusted, tunability over an order of magnitude in wavelength is possible. Second, with the use of an electro-magnet wiggler, it is possible to vary $\mathrm{K}$ to obtain rather fine tuning of the output wavelength. Finally, by using a carousel of wigglers whose spacings $\lambda_{w}$ cover a large range of values, it is possible to obtain very large variations in the optical wavelength.

The major advances in FEL technology have been in the generation and acceleration of the electron beam, the development of compact wigglers, and the development of control systems that permit low cost operation. Perhaps the most important of these is the beam generation aspect embodied in the development of the photo-electric injector whose operating principle is shown in Figure 3. The U-shaped cavity is representative of the input end of a high gradient accelerator cavity with a tri-alkali photo-cathode shown on the back surface of that cavity. At a selected time during the accelerator cavity's electric field sine wave, a laser pulse is used to illuminate the photo-cathode and release a burst of electrons. The pulse length of the electron beamlet is determined by the duration of the injection laser pulse, and is typically about 10 picoseconds. In addition, since the injection laser pulse (derived from a mode-locked neodymium-YLF laser) is synchronized with the rf pulse, the overall timing of the generation of the electron beam and its optimal injection into the cavity is assured. Figure 4 shows how the injector is used in the Los Alamos Advanced FEL (AFEL). It shows the photo-injector at the low energy end of an accelerator tube and the drive laser system that generates the electron beam via the photo-electric effect; note the absence of the modulator and buncher apparatus. Figure 5 shows graphically why the deveiopment of the photoelectric injector had such an impact on compact FELs. One of its inventors, Richard Sheffield, is shown standing behind the prototype model of the photo-injector system. Behind him is the electron gun and buncher system that typified FEL electron beam sources prior to the development of the photo-electric injector. 
The importance of keeping the emittance low cannot be over-emphasized and can be appreciated from Figure 6, the expression for single-pass gain in the laser section of the FEL taken from Datoli et al. We call attention to the terms in the denominator involving the quantities $\mathbf{m u}_{\mathbf{x}}$ and $\mathbf{m u}_{\mathbf{y}}$ which are the emittances in the direction transverse to the beam propagation direction. These enter quadratically and cause the gain of the laser to drop rapidly when they exceed 1 . The viability of small compact wigglers and efficient energy extraction from the electron beam depends on maintaining a very low emittance and a gain that exceeds the losses in the laser optical cavity.

We have used two methods to keep the emittance growth small. First, in a general sense, the electrons are bunched at their birth, and are accelerated to relativistic velocities in a matter of centimeters. The emittance of the beam remains low since the particles become rigid very quickly and the transverse spread due to space charge forces is kept small. Thus, the emittance growth produced by the need to bunch is simply absent. Second, we have reduced emittance growth in the low energy region near the cathode through the use of a solenoid, shown in Figure 3. At the cathode, the electron bunch emittance is determined by the cathode's thermal emitiance. As an electron bunch leaves the cathode, the bunch expands radially because of radial space charge forces. Since the space charge force acts continuously on the bunch, no single discrete lens can compensate for the resulting distortion of the beam's phase distribution. However, a simple lens can be used to focus the bunch. Then, to the first order, the same forces that acted on the bunch during expansion are present while the bunch is focused. Thus, the emittance growth that has occurred can be significantly reduced by proper lens placement. A unique solenoid design follows from the requirements of minimum emittance growth and simultaneously having the beam focused at a particular axial location, and depends on the accelerator gradient, current density, and location of the peak magnetic field with respect to the cathode. These methods have enabled us to produce meams with emittance below $5 \pi \mathrm{mm}-\mathrm{mr}$ at peak currents of $100-300$ amperes.

Another major advance in FEL technology is embodied in the utilization of very high gradient accelerator cavities. Figure 7 is a photograph of the cavity used for the AFEL prior to its encapsulation within bakeout coils used for ultra-high vacuum maintenance . It is approximately 1 meter long, and has an accelerating gradient of about 25 megavolts per meter. The energy gain from port to port is approximately 20 megavolts. The region closest to the observer in the figure is the point of attachment for the photo-electric injector system.

Because of the extremely low emittance and high peak current of the electron beam, it is possible to obtain adequate gain in a rather short wiggler ( 25 periods) Figure 8 is a photograph of the 25 centimeters long wiggler used in the AFEL. The magnetic field is obtained from rectangular Samarium-Cobalt magnets above and below the plane of the electron beam. At the center of the wiggler array, there is a shim with a central void, which is the channel for the electron beam and extends along the length of the wiggler. Although traditional wiggler designs have opposing poles transverse to the electron beam, the poles here are on the abutting faces of the magnet, i.e., in the plane of the electron beam. The repelling poles cause the magnetic field to dip down into the region of the electron beam and cause the undulation. 
A sophisticated diagnostic and control system is required for proper operation of the FEL. From the beginning, the AFEL was designed to have a computer controlled system in order to reduce its operating cost. Figure 9 shows the control console for the laser; it consists of a workstation and 3 partial racks of equipment which give the operator complete control over every aspect of the laser's operation, including its turn-on and turn-off functions.

Throughout the period of system design, assembly, and commissioning, simulation tools were absolutely essential. A variety of modeling and simulation programs were used. MAFIA, POISSON, and PARMELA were used to model the electric and magnetic fields and the propagation of the particles through them. The output of the PARMELA code feeds the laser simulator code, FELIX. The simulation process consists of birth to death tracking of thousands of particles, and includes the simulation of the diagnostics that are integral to the laser system; this is done in order to make sure that comparisons are done between relevant quantities--namely the predicted observables and the actual (not inferred) observables. The simulation codes have been heavily benchmarked and compared to all FEL systems for which we were able to obtain data. The codes provide a very reliable simulation method and design tool for complex systems such as FELs; in particular, the achievement of the low emittance described above would have been impossible without them.

One of the major advantages of the AFEL is its much smaller size; its compactness is best appreciated when compared to some FELs designed and built earlier. Figures 10 and 11 are photographs of the Los Alamos FEL, APEX, and the Boeing Aerospace FEL at Seattle as it existed about two years ago. Both systems have operated at average powers of a few watts (although the Boeing system was designed to operate at much higher powers), and utilized many thousands of square feet of floor space. Figure 12 is a line drawing of the AFEL with an outline of a person standing at the photo-injector end of the laser system. Its overall length is approximately 5 meters, its height is about 2 meters, and its depth is less than 1 meter. The system footprint area (including access and work space) is approximately two hundred square feet and represent an areal compaction of more than a factor of 50 compared to the systems of Figures 10 and 11. Figure 13 is a photograph of the assembled system; the size is difficult to appreciate because the small room in which the laser is housed precludes the taking of a side-on view of the system. The photo-electric injector is at the close end in the photograph; much of its complex plumbing is associated with the internal storage of spare photo-cathodes, the means for their replacement without breaking vacuum, and the need to maintain that region at ultra-high vacuum. The lifetime of the tri-alkali cathode in a quiescent mode is indefinite; in use, it is several days at efficiencies above $1 \%$. The accelerator is wrapped in insulated heating coils; its RF power is brought in via the duct shown in the upper left of the photograph. The wiggler and optical resonator are at the far end of the photograph. The spent-electron beam dump is buried in the concrete floor and can be seen as the dark opening in the floor at the far end of the laser system.

The design, development, assembly, and commissioning of the AFEL took approximately 3 years, and the full cost for the project was $\$ 5 \mathrm{M}$. This included all development and fabrication costs and the labor costs of assembling the system and rendering it operational. 
Eventually, production units are expected to cost significantly less--about $\$ 1-2 \mathrm{M}$. If, in addition, cathode materials can be found which are suitable for application in these systems and that do not require ultra-high vacuum operation, further reductions in cost and enhancement in utility would be possible.

In closing, we note a number of potential applications for a FEL that is compact, relatively inexpensive to operate, and low in acquisition cost. These applications are shown in Figures 14 - 16. The first of these is a concept for an FEL extreme ultraviolet light source. The system consists of a photo-electric injector, four 25 megavolt accelerating cavity modules to give a total energy of 100 megavolts, a bending station to reverse the direction of the electron beam, and a resonator system that utilizes mirrors based on the principle of total external reflection. The low emittance of the electron beam permits the direct generation of light down to a wavelength of approximately 50 nanometers; even shorter wavelengths are possible with higher beam energies. The other applications include use of FELs for near field microscopy and mapping macro molecules, based on techniques developed by Bob Austin of Princeton; the use of FELs in atmospheric science for LIDAR, to measure wind patterns in the atmosphere, to measure greenhouse gases, and as a probe to provide atmospheric compensation for light beam propagation.

Finally, we envision applications for the uses of FELs as "enabling tools": cutting and coagulation of tissue (or at least to determine the best optical wavelength for such applications), cutting and welding in industrial applications, and as a light source "catalyst" for photo-chemical processing. Conceivably, one of the best applications of the AFEL will be in intense tunable light sources for material science research. 


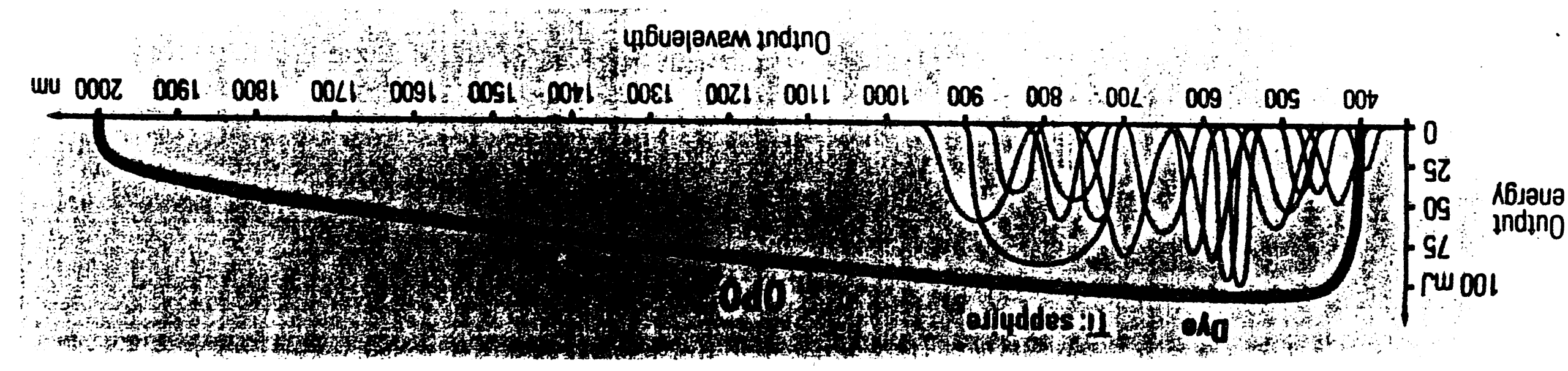

\section{(К‼!qejeos pue) ләмод әбеләл $\forall$ 'ueds Чұбиәрәлем

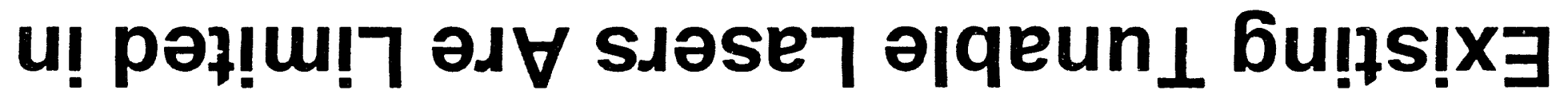




\section{The Basic Free-Electron Laser}

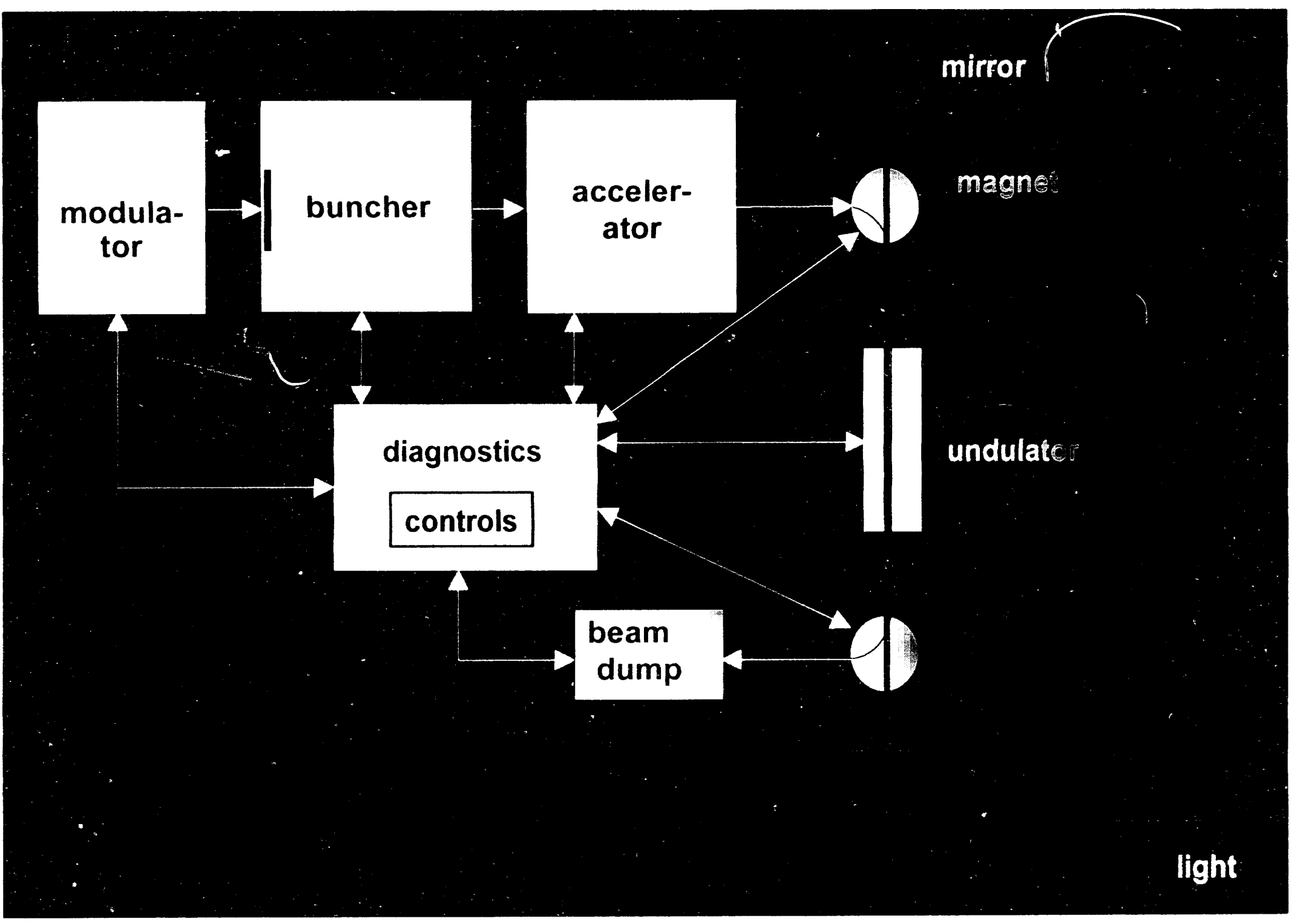




\section{Photoinjector Produces Bright, Low-Emittance Beams}

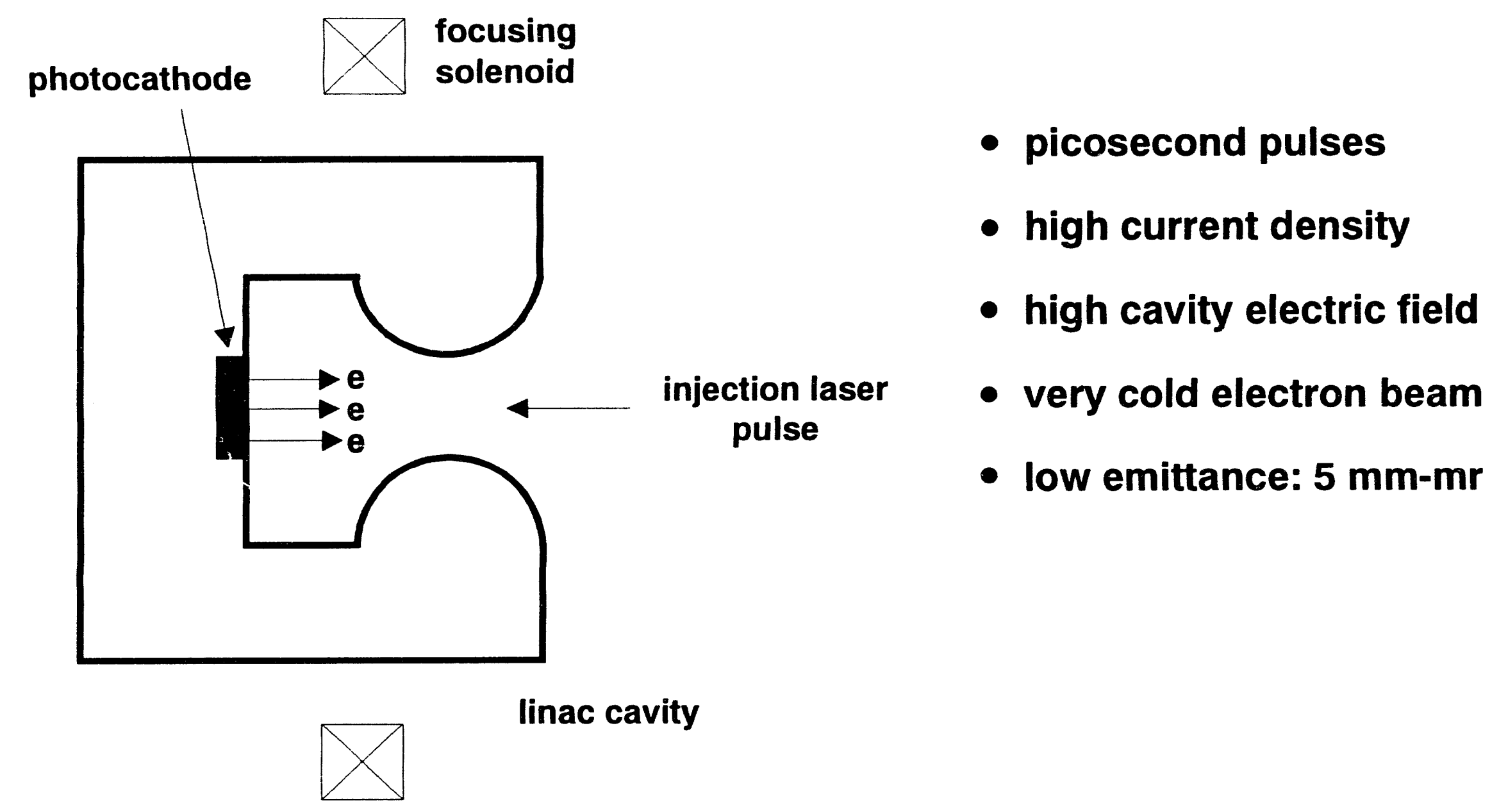




\section{The Advanced Free-Electron Laser}

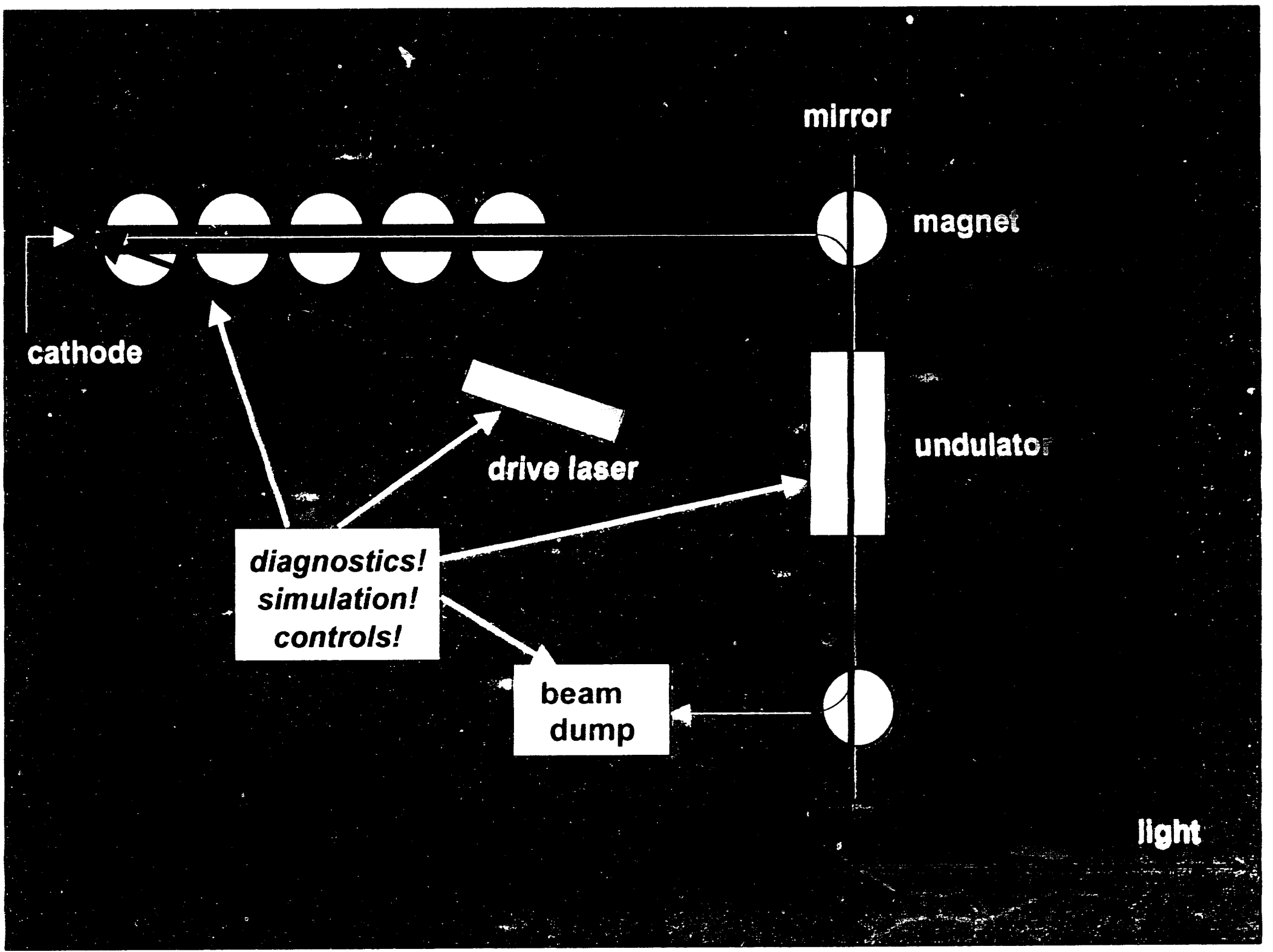


pras

A. $-x$

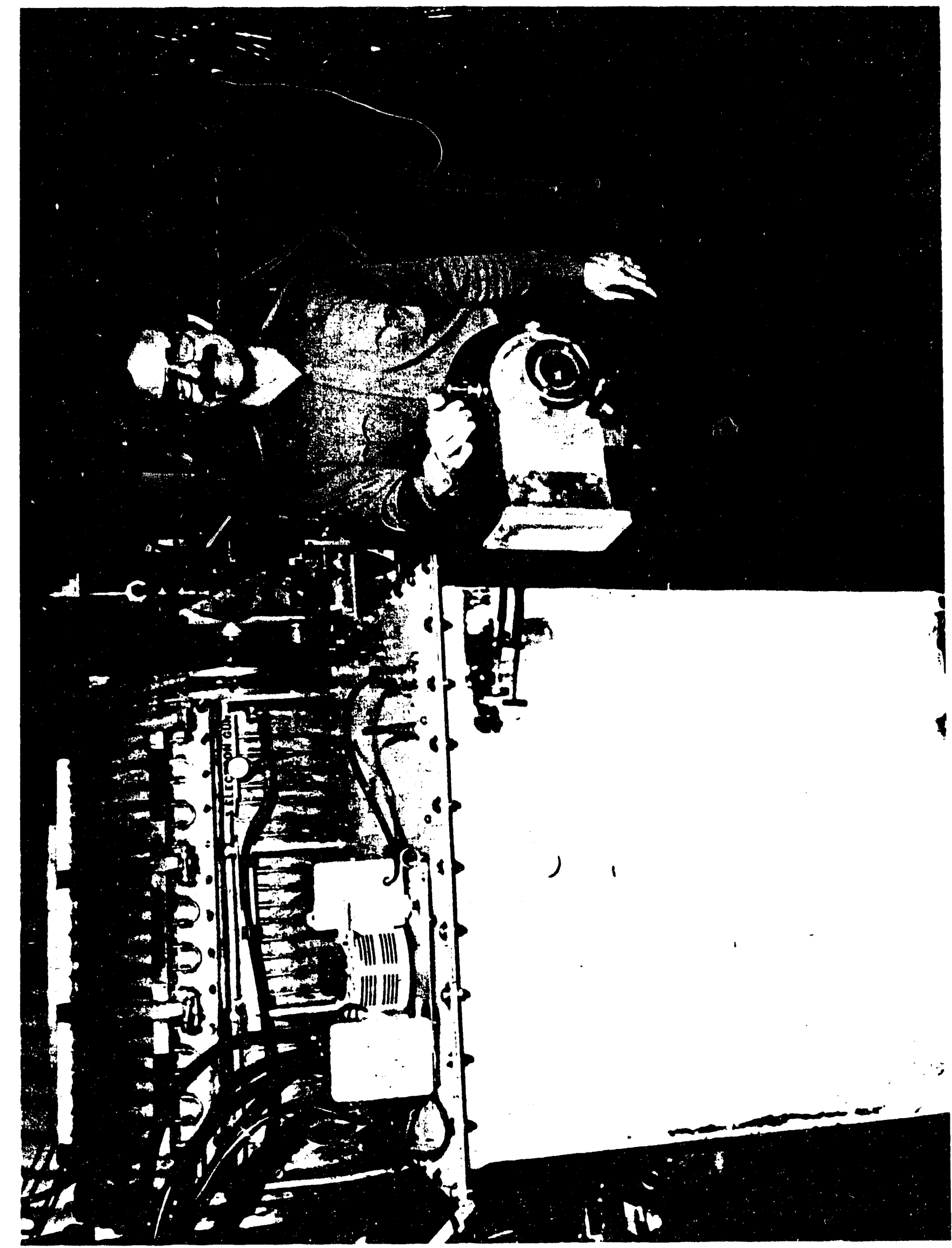




\section{Emittance, Energy Spread: Critical Parameters}

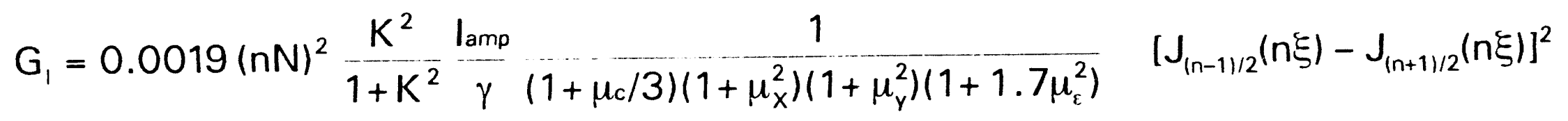

$\mathrm{n}, \mathrm{N}=$ harmonic number, number of undulator elements

$\mathrm{K}=$ undulator parameter, $\frac{\mathrm{eB} \lambda_{a}}{\sqrt{8 \pi m c^{2}}}$

$\mu_{c}=$ coupling parameter, $\frac{\lambda_{0}}{2 \sigma_{z}}\left[\frac{\omega}{\Delta \omega}\right]_{0}$

$\mu_{x, y}=\sqrt{2 h_{x, y}} \frac{K}{1+K^{2}} \frac{\gamma \varepsilon_{x, y}}{\lambda_{a}}\left[\frac{\omega}{\Delta \omega}\right]_{0}$

$\mu_{\varepsilon}=2 \sigma_{e}\left[\frac{\omega}{\Delta \omega}\right]_{0}$

$\xi=\frac{K^{2}}{2\left(1+K^{2}\right)}$ 


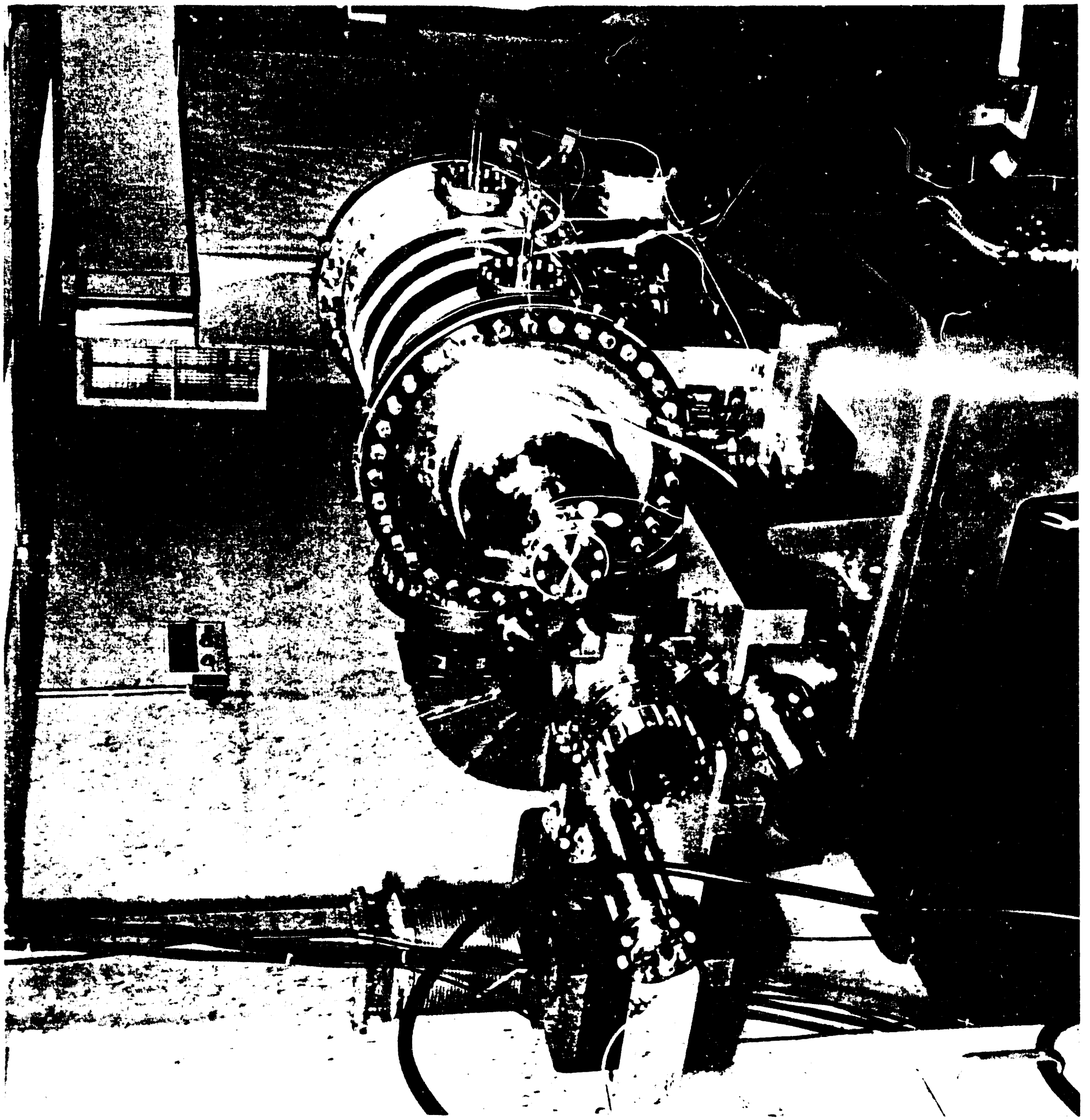




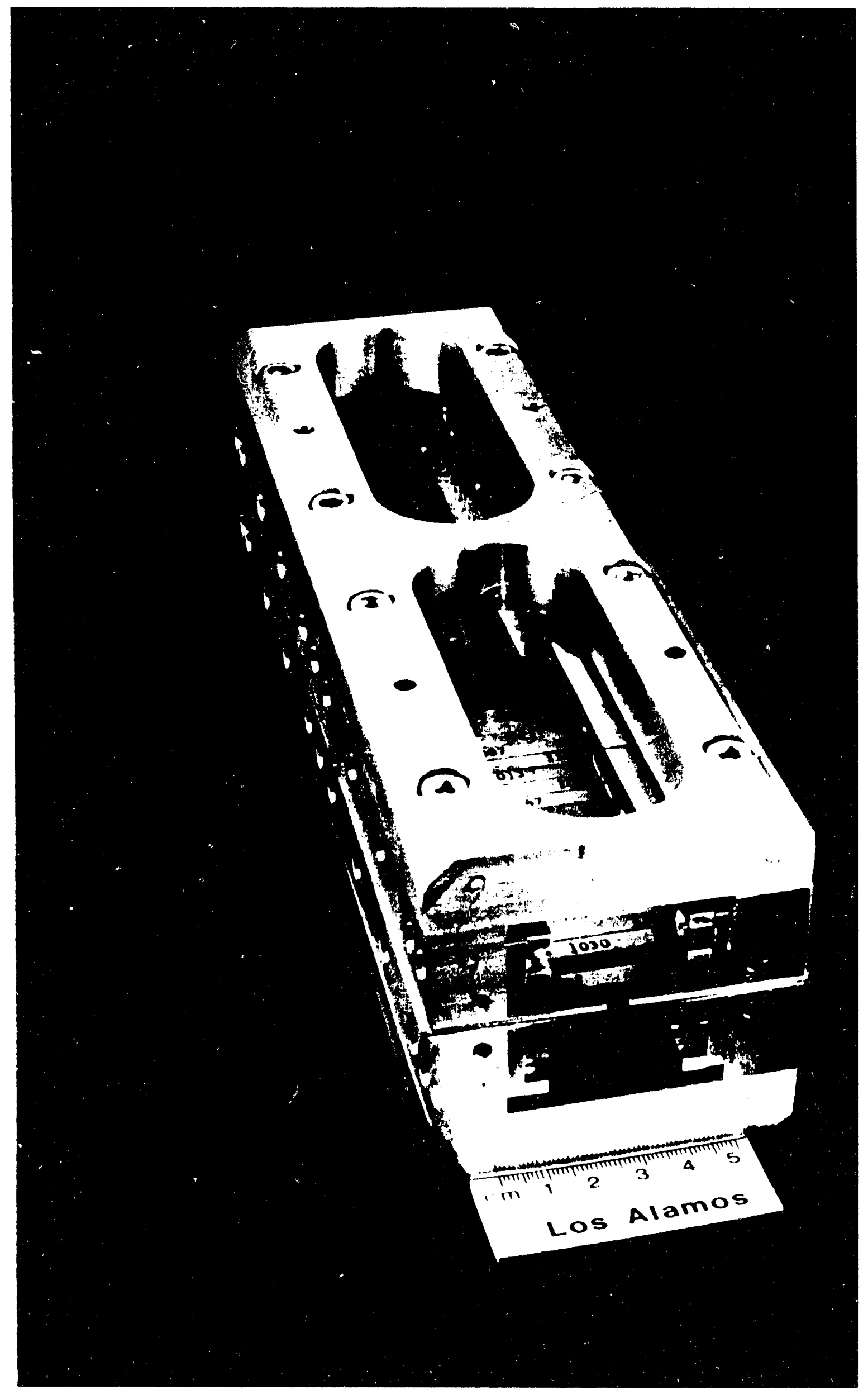




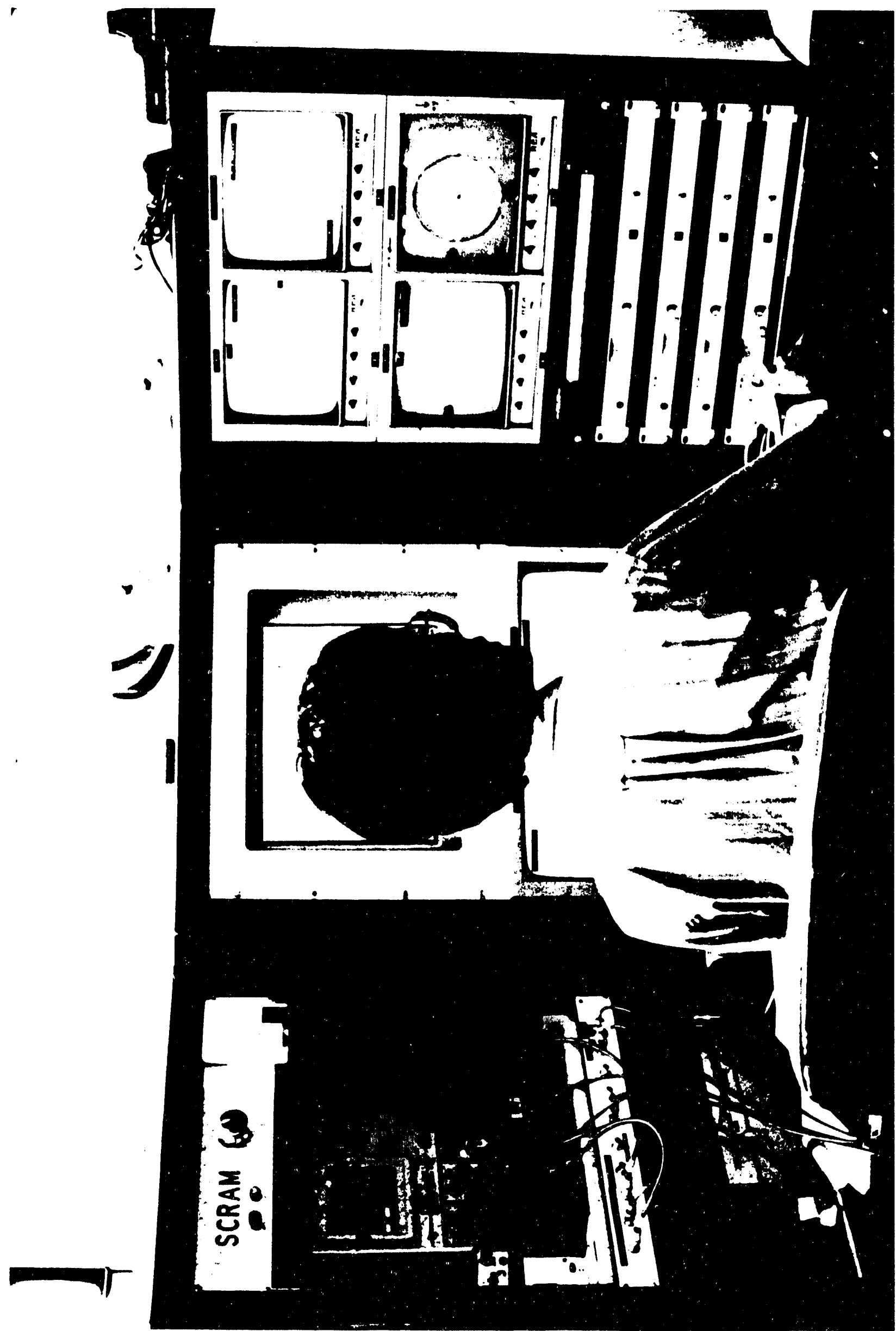




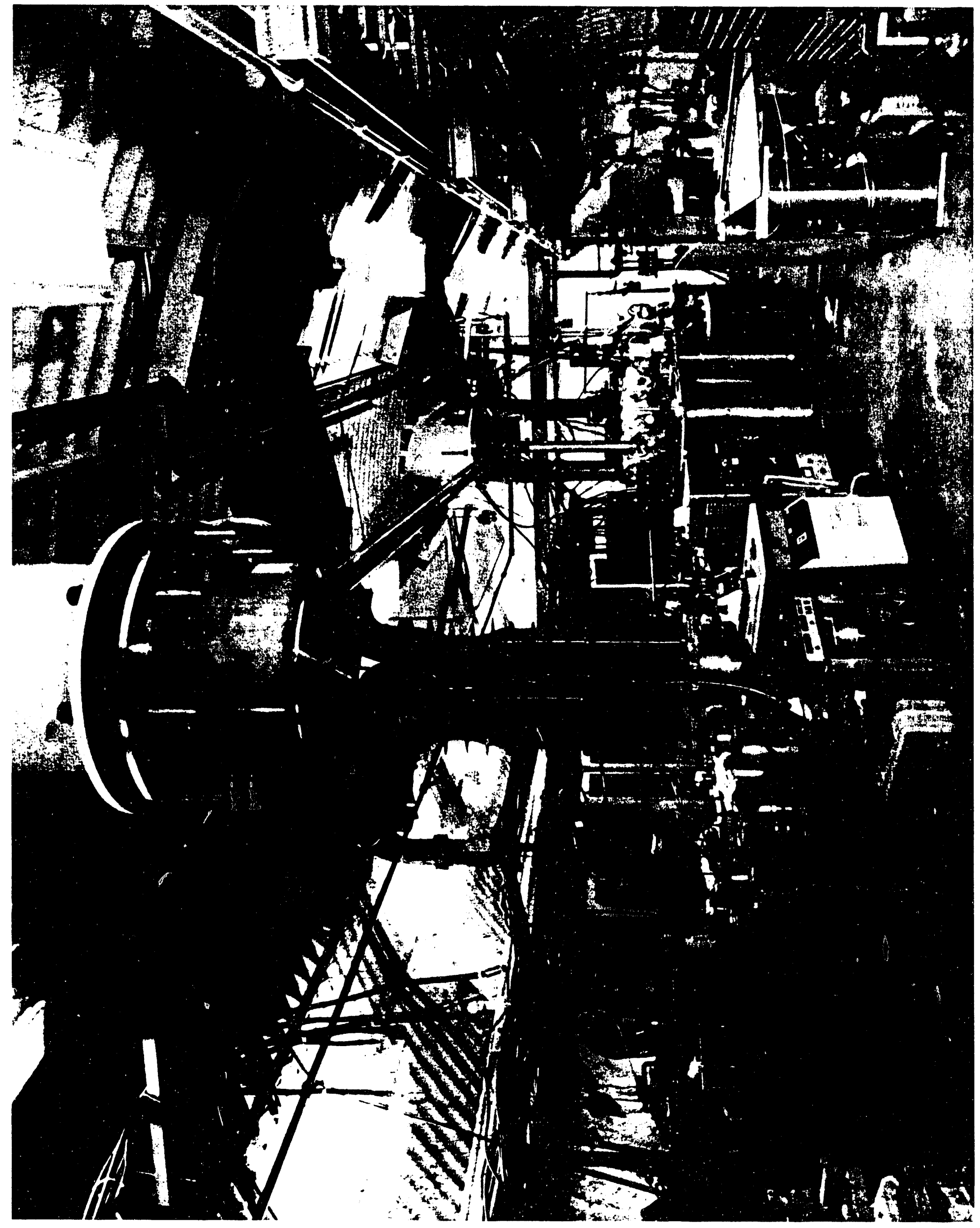




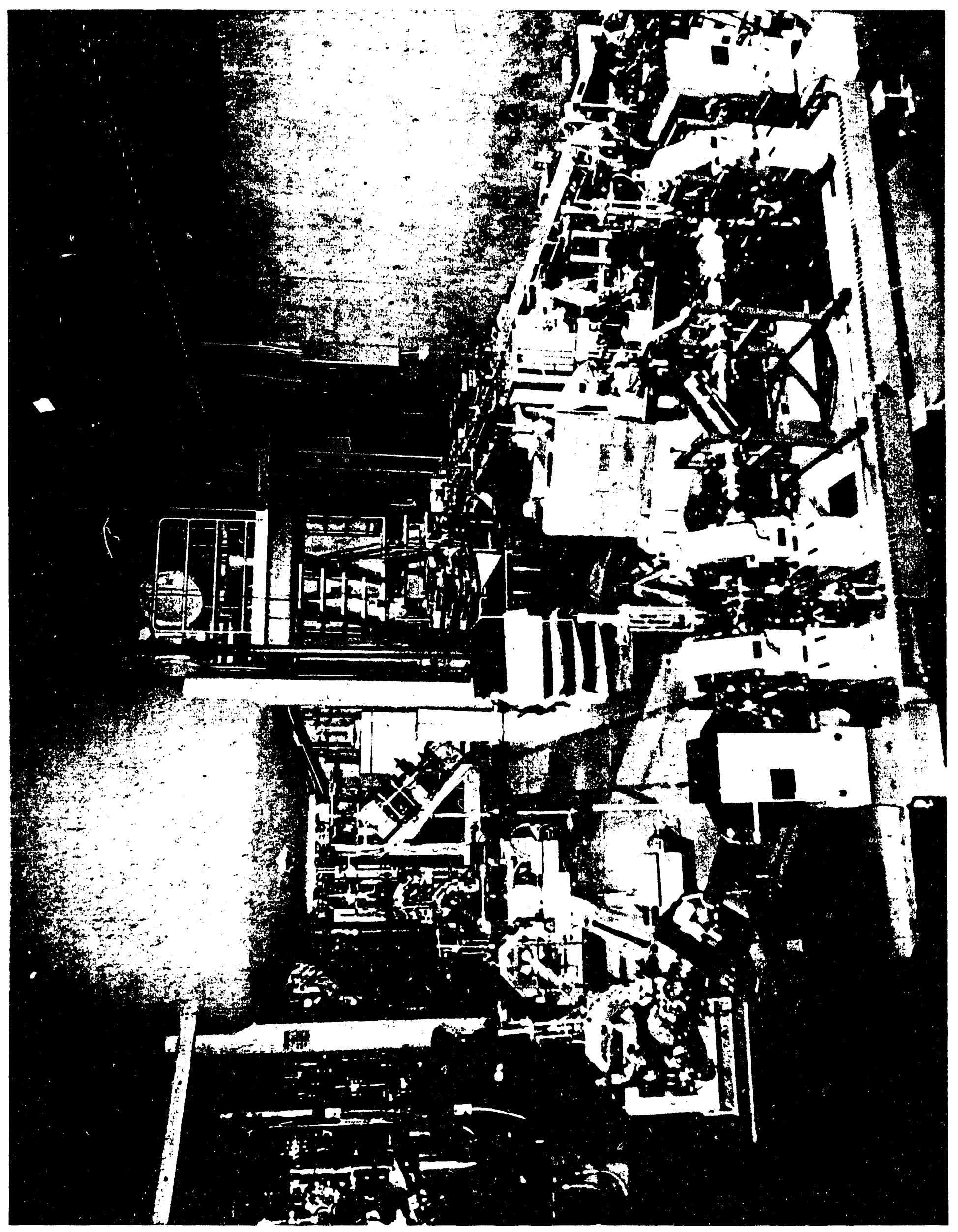




\section{THE AFEL MACHINE IS COMPACT AND WILL PRODUCE A VERY BRIGHT ELECTRON BEAM}

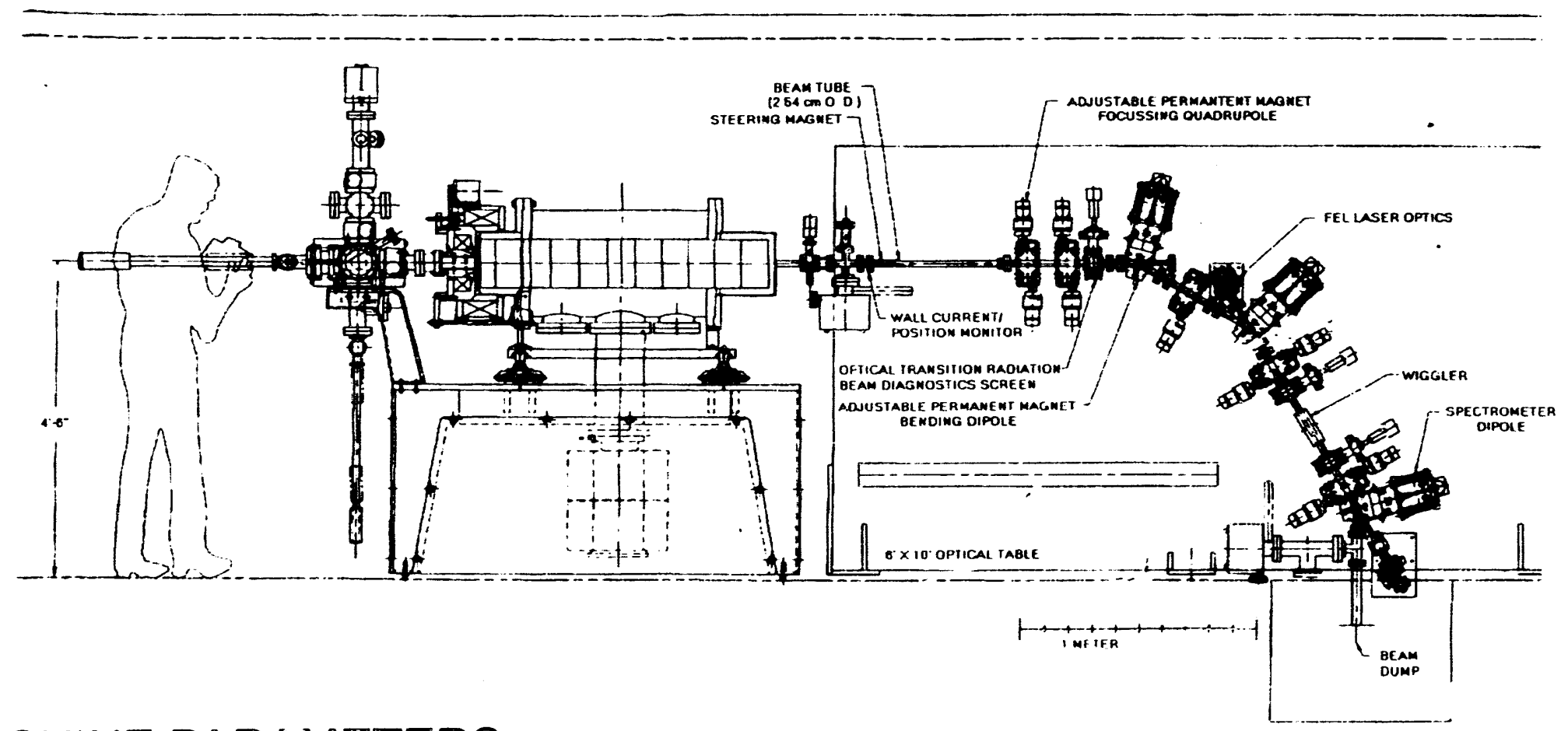

MACHINE PARAMETERS:

ENERGY: $20 \mathrm{MeV}$

ENERGY SPREAD: $<0.2 \%$

CURRENT: 200 to 300 A peak, 10 ps micropulse $10 \mu s$ macropulse, $10 \mathrm{~Hz}$ EMITTANCE: $<15 \pi \mathrm{mm}$-mrad for whole pulse, $<2 \pi \mathrm{mm}$-mrad for middle $75 \%$ 
$E$
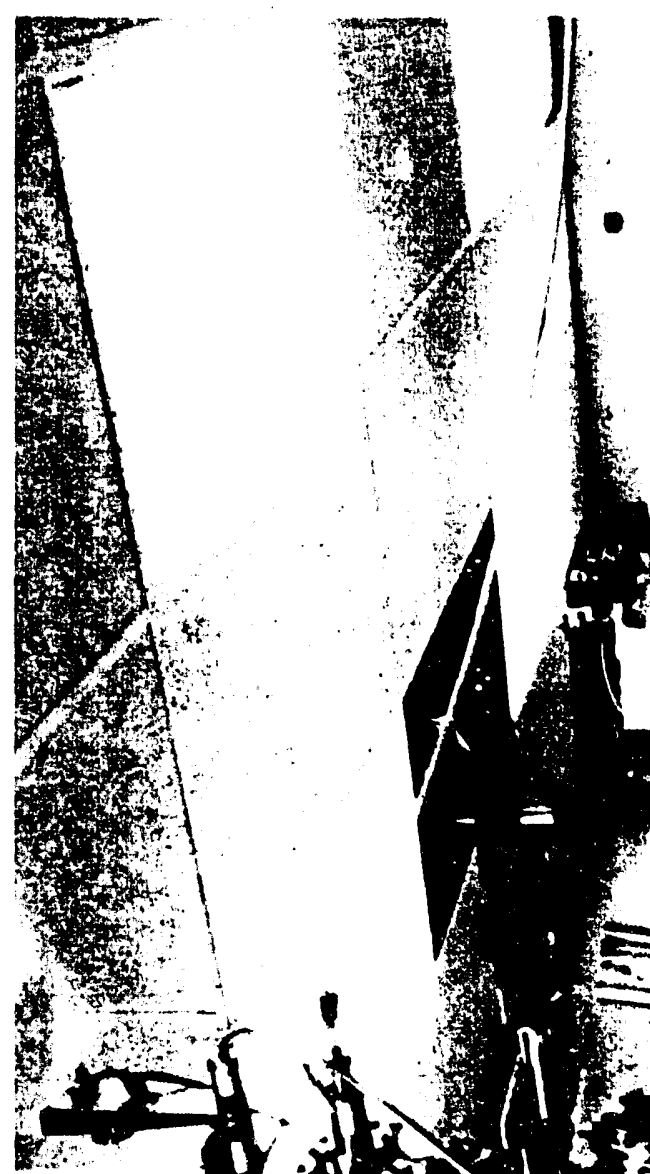

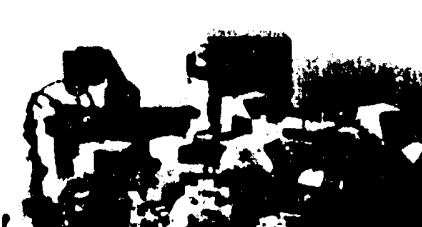

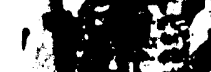

$\rightarrow 0$

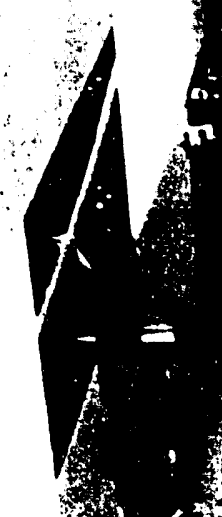

a
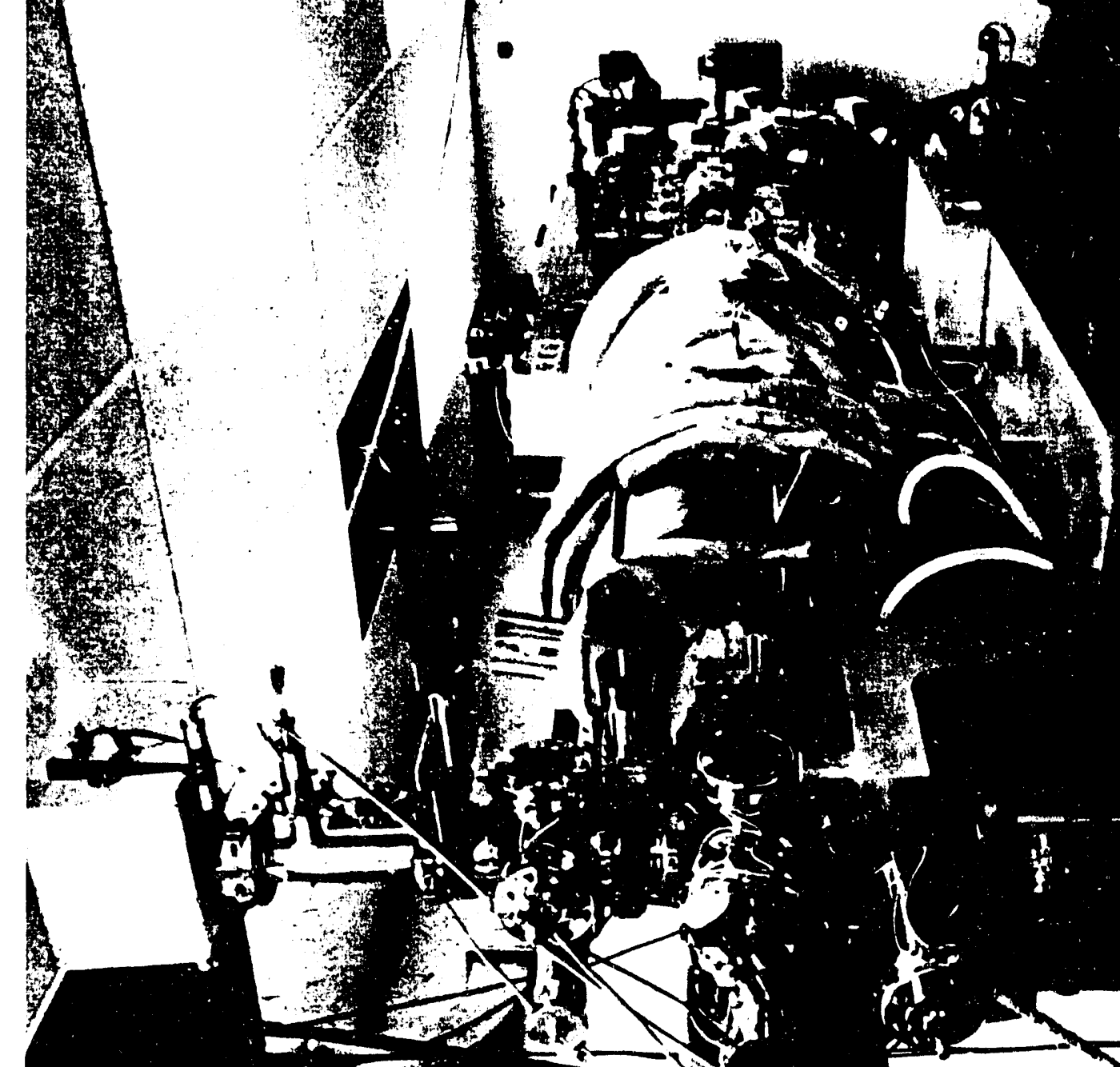

. $1 / 4 d$ . 1 iv Ex 1 5. 40

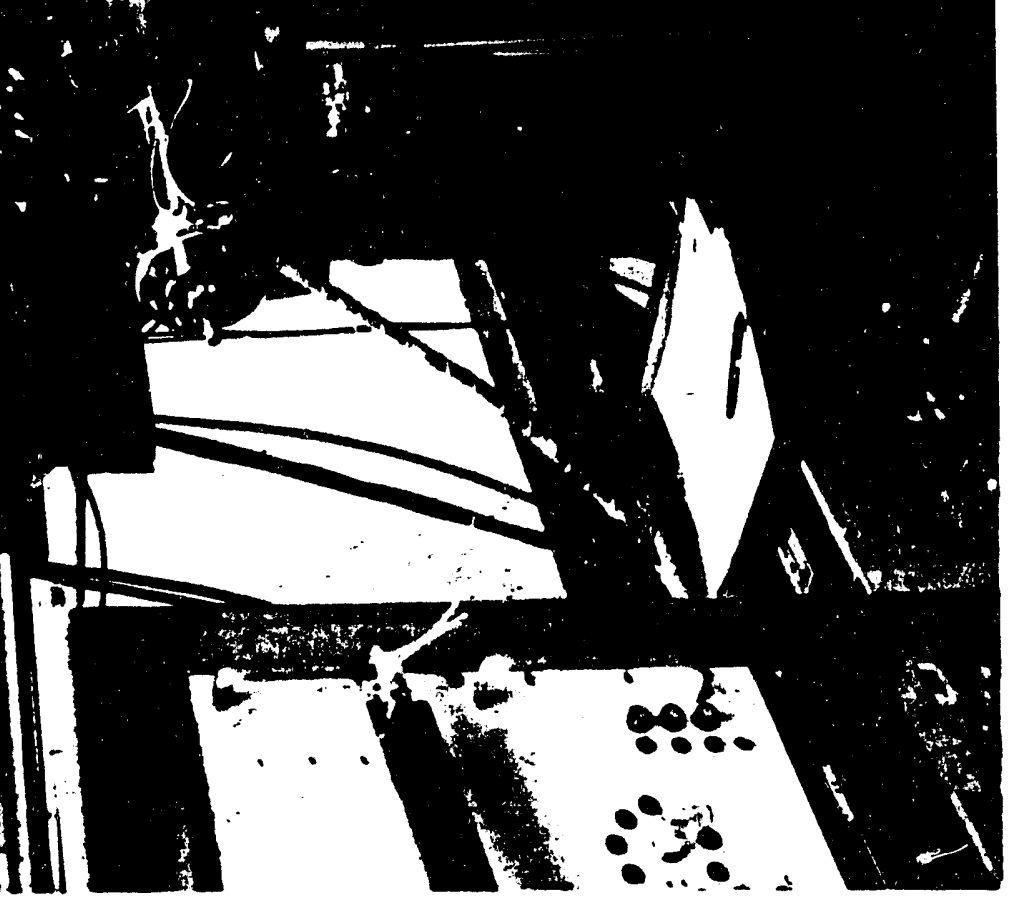




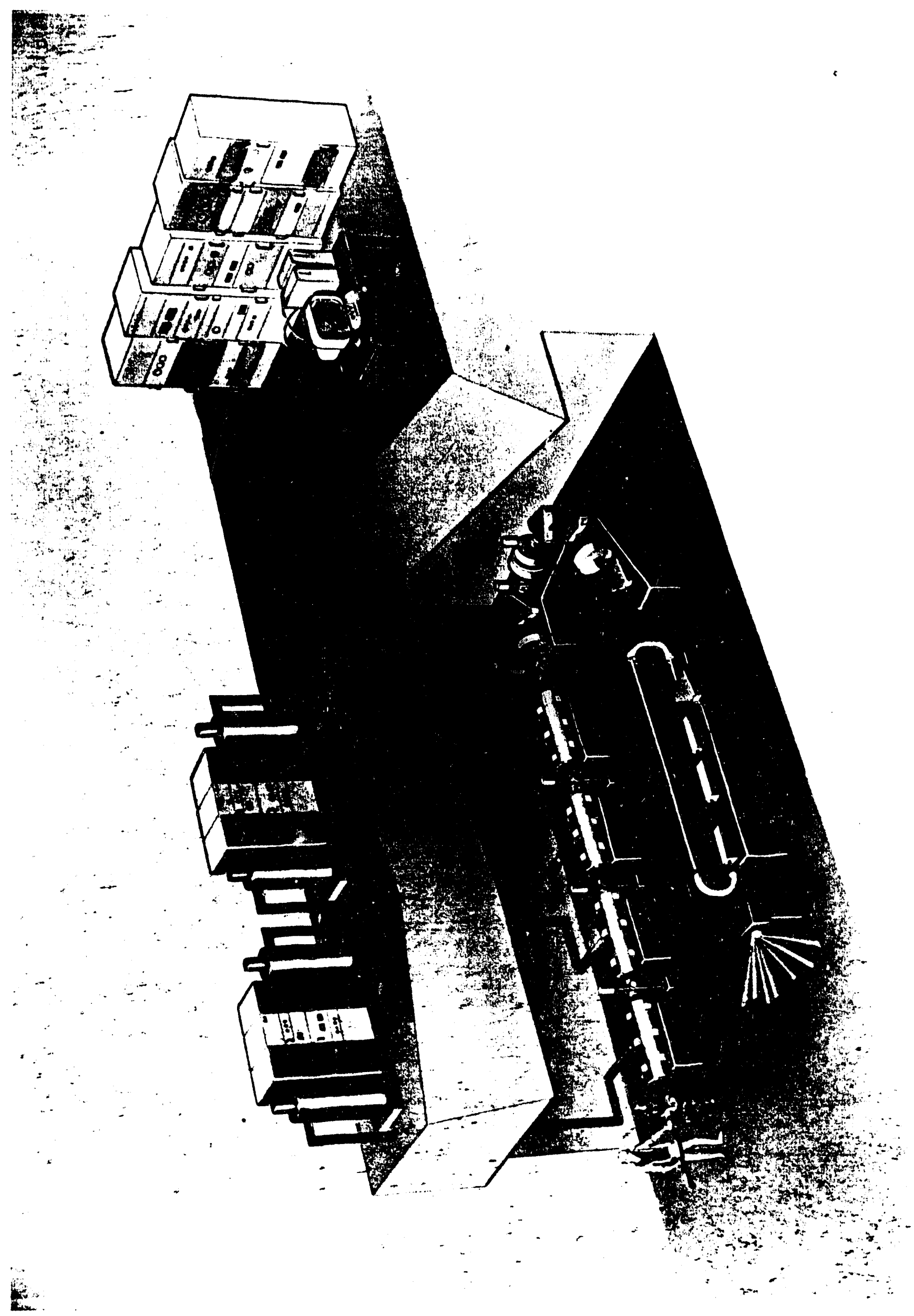




\section{LIFE SCIENCE}

Basic Application:

Near field microscopy for physical mapping of macromolecules (DNA, proteins, etc.)

Experiment based on FEL operation in the infrared with STM and nanolithography tools supplied by Professor Robert Auston (on sabbatical leave from Princeton).

NEAR-FIELD

MICROSCOPY

WITH OPTICAL

MICROPROBE

FEL BEAM

LIGHT PIPE

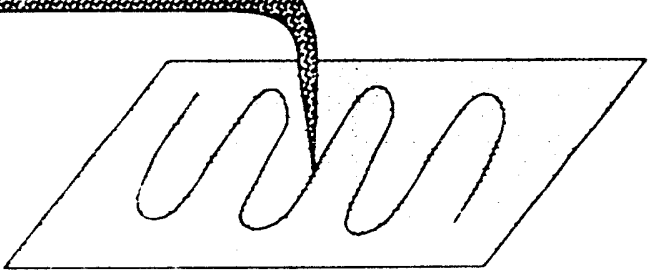

DNA OR MACROMOLECULES ON 2-D LITHOGRAPHIC PATTERNS 


\section{ATMOSPHERIC SCIENCE}

Basic applications:

- LIDAR (for wind patterns in the upper atmosphere)

- DIAL (to measure greenhouse gases)

- atmospheric compensation (correction for atmospheric turbulence).

We will do a test for differential absorption LIDAR (DIAL). We will operate at $2.7 \mu \mathrm{m}$ and $4.1 \mu \mathrm{m}$ to make measurements of $\mathrm{H}_{2} \mathrm{O}$ and $\mathrm{CO}_{2}$ at TA-53.

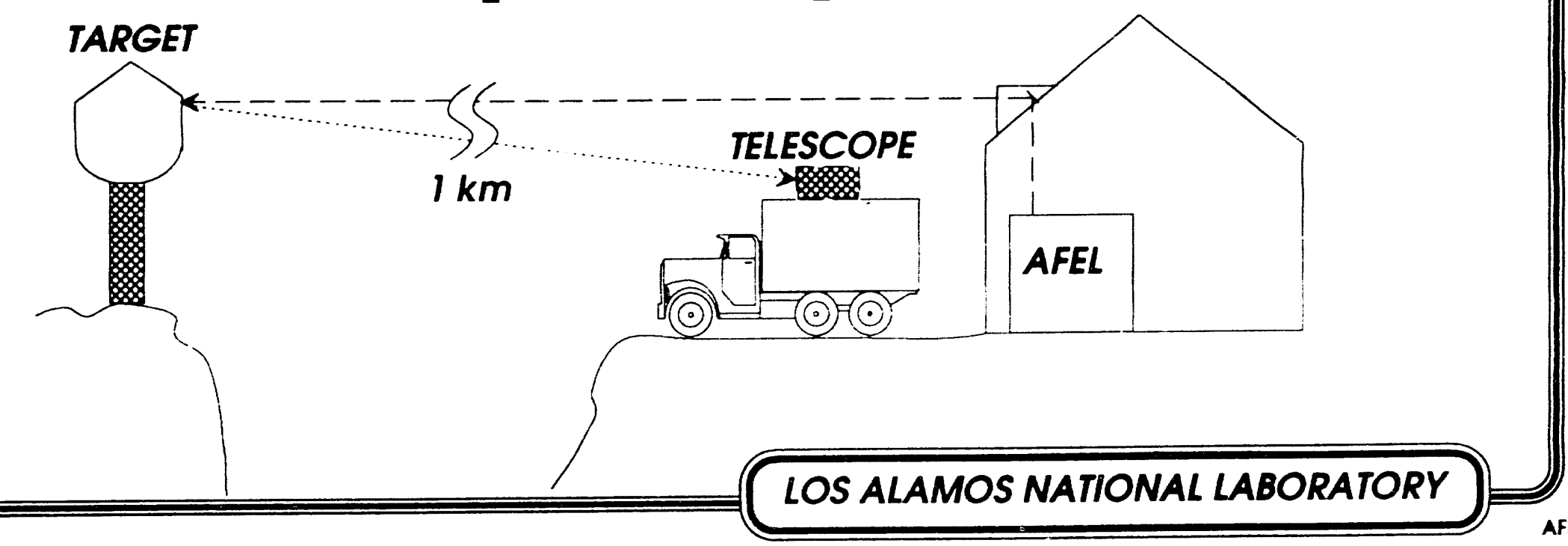




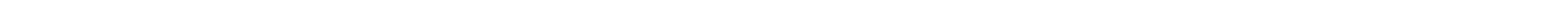


